Volume 70, Number 1, Pages 74-81(2021)

DOI: $10.31801 /$ cfsuasmas.710601

ISSN 1303-5991 E-ISSN 2618-6470

Received by the editors: March 28, 2020; Accepted: November 25, 2020

\title{
ON ALMOST HUREWICZ PROPERTY IN BITOPOLOGICAL SPACES
}

\author{
Harsh V. S. CHAUHAN and Beenu SINGH
}

Department of Mathematics, University of Delhi, New Delhi-110007, INDIA

\begin{abstract}
The purpose of this paper is to investigate properties and results of the almost Hurewicz property in bitopological spaces. We characterize this property using $(i, j)$ - regular open sets and almost continuous surjective maps.
\end{abstract}

\section{INTRODUCTION}

In topology, covering properties have been studied by many authors such as K. Menger [16], W. Hurewicz [5, 6, F. Rothberger 20]. In 1925, W. Hurewicz introduced the concept of Hurewicz property. A space $X$ is defined to be Hurewicz if for each sequence $\left(\mathcal{U}_{n}: n \in \mathbb{N}\right)$ of open covers of $X$, there exists a sequence $\left(\mathcal{V}_{n}: n \in \mathbb{N}\right)$ such that for each $n \in \mathbb{N}, \mathcal{V}_{n}$ is a finite subset of $\mathcal{U}_{n}$ and for each $x \in X, x \in \bigcup\left\{V: V \in \mathcal{V}_{n}\right\}$ for all but finitely many $n$.

In 1996, Scheepers 22 introduced the theory of selection principles by using selection hypothesis to the different types of open covers. The selection principles have many applications in daily life and mainly in game theory. In recent years, many generalizations and weaker forms of Hurewicz space like almost Hurewicz space, weak Hurewicz space have been come into the picture and studied [10,21, 24 25].

In the last few years, covering properties related to selective versions of separability and selection principle in function spaces are studied in bitopological spaces [8, 11, 14, 17, 18. The concepts of almost Menger property, alomst Rothberger property and almost $\gamma$-sets in bitopological sense, have been introduced in [13 19]. In 2013, Y. K. Song et al. 24] demonstrated almost Hurewicz property in detail. Recently, in [4], Eysen et al. studied weaker forms of Menger property

2020 Mathematics Subject Classification. 54D20, 54E55.

Keywords and phrases. Hurewicz property, almost Hurewicz property, bitopological space, Menger property.

\harsh.chauhan111@gmail.com; singhbeenu47@gmail.com-Corresponding author

(D) 0000-0002-0888-4221; 0000-0003-0196-7670.

(C)2021 Ankara University Communications Faculty of Sciences University of Ankara-Series A1 Mathematics and Statistics 
in bitopological spaces and also considered almost Hurewicz property in bitopological spaces. In the present paper, we are concerned to study several properties and results of the almost Hurewicz spaces in bitopological context. Section 2 contains some basic definitions and results. In Section 3, almost Hurewicz property in bitopological spaces is defined, and it is shown that if $(i, j)$-almost Hurewicz space $X$ is $(i, j)$-regular, then each topological space $\left(X, \mathcal{T}_{i}\right)$ where $i=1,2$ is Hurewicz. Some characterizations with necessary and sufficient conditions for $(i, j)$-almost Hurewicz spaces are substantiated.

\section{Preliminaries}

Definition 2.1. 24] A topological space $X$ is called almost Hurewicz if for each sequence $\left(\mathcal{U}_{n}: n \in \mathbb{N}\right)$ of open covers of $X$ there is a sequence $\left(\mathcal{V}_{n}: n \in \mathbb{N}\right)$ such that for each $n \in \mathbb{N}, \mathcal{V}_{n}$ is a finite subset of $\mathcal{U}_{n}$ and for each $x \in X, x \in \bigcup\{C l(V)$ : $\left.V \in \mathcal{V}_{n}\right\}$ for all but finitely many $n$.

Definition 2.2. [23] Let $\left(X, \mathcal{T}_{1}, \mathcal{T}_{2}\right)$ be a bitopological space. Then it is called $(i, j)$ regular where $i, j=1,2$ and $i \neq j$ if for each $x \in X$ and each $\mathcal{T}_{i}$-closed set $F$ of $X$ with $x \notin F$, there exists a $\mathcal{T}_{i}$-open set $U$ containing $x$ and a $\mathcal{T}_{j}$-open set $W$ containing $F$ such that $U \cap W=\emptyset$.

Theorem 2.3. 23] Let $\left(X, \mathcal{T}_{1}, \mathcal{T}_{2}\right)$ be a bitopological space. Then $X$ is $(i, j)$-regular if and only if for each $x \in X$ and each $\mathcal{T}_{i}$-open set $U$ containing $x$, there exists $\mathcal{T}_{i}$ open set $W$ such that $x \in W \subseteq C l_{\mathcal{T}_{j}}(W) \subseteq U$.

Definition 2.4. [9, 23] Let $\left(X, \mathcal{T}_{1}, \mathcal{T}_{2}\right)$ be a bitopological space and $A \subseteq X$. Then $A$ is said to be $(i, j)$-regular open (or $(i, j)$-regular closed) where $i, j=1,2$ and $i \neq j$, if $A=\operatorname{int}_{\mathcal{T}_{i}} C l_{\mathcal{T}_{j}}(A)$ (or $\left.A=C l_{\mathcal{T}_{i}} i_{\mathcal{T}_{j}}(A)\right)$. The set $A$ is pairwise regular open (or pairwise regular closed) if it is both $(i, j)$-regular open and $(j, i)$-regular open (or $(i, j)$-regular closed and $(j, i)$-regular closed $)$.

Definition 2.5. [15] A map $f:\left(X, \mathcal{T}_{1}, \mathcal{T}_{2}\right) \longrightarrow\left(Y, \mathcal{T}_{1}^{\prime}, \mathcal{T}_{2}^{\prime}\right)$ is said to be $(i, j)$-almost continuous if $f^{-1}(U)$ is $\mathcal{T}_{i}$-open set in $X$ for every $(i, j)$-regular open set $U$ in $Y$. Moreover, $f$ is p-almost continuous if it is $(1,2)$ - and $(2,1)$-almost continuous.

Definition 2.6. [4] Let $\left(X, \mathcal{T}_{1}, \mathcal{T}_{2}\right)$ be a bitopological space. Then $X$ is said to be $d$-compact if it is both $\mathcal{T}_{1}$-compact and $\mathcal{T}_{2}$-compact.

Definition 2.7. [2] Let $\left(X, \mathcal{T}_{1}, \mathcal{T}_{2}\right)$ and $\left(Y, \mathcal{T}_{1}^{\prime}, \mathcal{T}_{2}^{\prime}\right)$ be the bitopological spaces. Then a mapping $f:\left(X, \mathcal{T}_{1}, \mathcal{T}_{2}\right) \longrightarrow\left(Y, \mathcal{T}_{1}^{\prime}, \mathcal{T}_{2}^{\prime}\right)$ is said to be $i$-continuous $(i$-closed) if the induced mappings $f:\left(X, \mathcal{T}_{i}\right) \longrightarrow\left(Y, \mathcal{T}_{i}^{\prime}\right)$ are continuous (closed).

Definition 2.8. [4] A mapping $f:\left(X, \mathcal{T}_{1}, \mathcal{T}_{2}\right) \longrightarrow\left(Y, \mathcal{T}_{1}^{\prime}, \mathcal{T}_{2}^{\prime}\right)$ is said to be $d$ continuous (d-closed) if it is 1-continuous and 2-continuous.

Definition 2.9. [1] Let $\left(X, \mathcal{T}_{1}, \mathcal{T}_{2}\right)$ and $\left(Y, \mathcal{T}_{1}^{\prime}, \mathcal{T}_{2}^{\prime}\right)$ be the bitopological spaces. Then a d-continuous, d-closed mapping $f: X \rightarrow Y$ is called perfect if $\forall y \in Y$, the set $f^{-1}(y)$ is d-compact in $X$. 
Definition 2.10. [7] A mapping $f:\left(X, \mathcal{T}_{1}, \mathcal{T}_{2}\right) \longrightarrow\left(Y, \mathcal{T}_{1}^{\prime}, \mathcal{T}_{2}^{\prime}\right)$ is said to be $(i, j)$ preopen if $f(U) \subset$ int $_{\mathcal{T}_{i}^{\prime}} C l_{\mathcal{T}_{j}^{\prime}}(f(U))$ for each $\mathcal{T}_{i}$-open subset $U$ of $X$.

Proposition 2.11. [4] A mapping $f:\left(X, \mathcal{T}_{1}, \mathcal{T}_{2}\right) \longrightarrow\left(Y, \mathcal{T}_{1}^{\prime}, \mathcal{T}_{2}^{\prime}\right)$ is $(i, j)$-preopen if and only if $f^{-1}\left(C l_{\mathcal{T}_{i}^{\prime}}(U)\right) \subset C l_{\mathcal{T}_{i}}\left(f^{-1}(V)\right)$ for each $\mathcal{T}_{j}^{\prime}$-open subset $V$ of $Y$.

Definition 2.12. [19] Let $\left(X, \mathcal{T}_{1}, \mathcal{T}_{2}\right)$ be a bitopological space. Then it is called $(i, j)$-almost Menger if for each sequence $\left(\mathcal{U}_{n}: n \in \mathbb{N}\right)$ of $\mathcal{T}_{i}$ - open covers of $X$, there exists a sequence $\left(\mathcal{V}_{n}: n \in \mathbb{N}\right)$ such that for each $n \in \mathbb{N}, \mathcal{V}_{n}$ is a finite subset of $\mathcal{U}_{n}$ and $X=\bigcup_{n \in \mathbb{N}}\left(\bigcup_{V \in \mathcal{V}_{n}} C l_{\mathcal{T}_{j}}(V)\right)$.

\section{3. $(i, j)$-Almost Hurewicz Property}

Definition 3.1. Let $\left(X, \mathcal{T}_{1}, \mathcal{T}_{2}\right)$ be a bitopological space. Then $X$ is said to be $(i, j)$ almost Hurewicz if for each sequence $\left(\mathcal{U}_{n}: n \in \mathbb{N}\right)$ of $\mathcal{T}_{i}$ - open covers of $X$, there exists a sequence $\left(\mathcal{V}_{n}: n \in \mathbb{N}\right)$ such that for each $n \in \mathbb{N}, \mathcal{V}_{n}$ is a finite subset of $\mathcal{U}_{n}$ and for each $x \in X, x \in \bigcup\left\{C l_{\mathcal{I}_{j}}(V): V \in \mathcal{V}_{n}\right\}$ for all but finitely many $n$.

Obviously, every $(i, j)$-almost Hurewicz space is $(i, j)$-almost Menger. Note that if $\left(X, \mathcal{T}_{1}\right)$ is almost Hurewicz space and $\mathcal{T}_{2}$ is a topology on $X$ such that $\mathcal{T}_{1} \geq \mathcal{T}_{2}$, that is, $\mathcal{T}_{2}$ is coarser than $\mathcal{T}_{1}$, then the bitopological space $\left(X, \mathcal{T}_{1}, \mathcal{T}_{2}\right)$ is $(1,2)$-almost Hurewicz.

Lemma 3.2. If $\left(X, \mathcal{T}_{1}\right)$ is a Hurewicz space, then the bitopological space $\left(X, \mathcal{T}_{1}, \mathcal{T}_{2}\right)$ is $(1,2)$-almost Hurewicz.

The converse of above lemma need not be true in general as shown in next example.

Example 3.3. Let $X=\mathbb{R}^{2}$ with $\mathcal{T}_{1}=\mathbb{R}_{l} \times \mathbb{R}_{l}$, where $R_{l}$ be a lower limit topology on $\mathbb{R}$ and $\mathcal{T}_{2}$ be usual topology on $X$. Then $\left(X, \mathcal{T}_{1}, \mathcal{T}_{2}\right)$ is $(1,2)$-almost Hurewicz. But $\left(X, \mathcal{T}_{1}\right)$ is not Hurewicz.

Now in the following theorem we conclude that the converse of Lemma 3.2 is true if $X$ is $(i, j)$-regular.

Theorem 3.4. Let $\left(X, \mathcal{T}_{1}, \mathcal{T}_{2}\right)$ be a bitopological space. If $X$ is $(i, j)$-almost Hurewicz and $(i, j)$-regular, then $\left(X, \mathcal{T}_{i}\right)$ is a Hurewicz space.

Proof. Let $i=1$ and $j=2$. Let $\left(\mathcal{U}_{n}: n \in \mathbb{N}\right)$ be a sequence of $\mathcal{T}_{1}$ - open covers of $X$. Since $X$ is an $(1,2)$-regular space, therefore for each $n \in \mathbb{N}$ there exists a $\mathcal{T}_{1}$ open cover $\mathcal{W}_{n}$ such that $C l_{\mathcal{T}_{2}}\left(\mathcal{W}_{n}\right)$ is a refinement of $\mathcal{U}_{n}$. Also as $X$ is $(1,2)$-almost Hurewicz, there exists a sequence $\left(\mathcal{W}_{n}^{\prime}: n \in \mathbb{N}\right)$ such that for each $n \in \mathbb{N}, \mathcal{W}_{n}^{\prime}$ is a finite subset of $\mathcal{W}_{n}$ and for each $x \in X, x \in \bigcup\left\{C l_{\mathcal{T}_{2}}(W): W \in \mathcal{W}_{n}^{\prime}\right\}$ for all but finitely many $n$. Thus, for any $n \in \mathbb{N}$ and $W^{\prime} \in \mathcal{W}_{n}^{\prime}$, choose $U_{W^{\prime}} \in \mathcal{U}_{n}$ such that $C l_{\mathcal{T}_{2}}\left(W^{\prime}\right) \subseteq U_{W^{\prime}}$. Now let $\mathcal{V}_{n}=\left\{U_{W^{\prime}}: W^{\prime} \in \mathcal{W}_{n}^{\prime}\right\}$; then $\left(\mathcal{V}_{n}: n \in \mathbb{N}\right)$ is a sequence such that for each $n \in \mathbb{N}, \mathcal{V}_{n}$ is a finite subset of $\mathcal{U}_{n}$ and for each $x \in X, x \in \bigcup \mathcal{V}_{n}$ for all but finitely many $n$. Hence, $\left(X, \mathcal{T}_{i}\right)$ is Hurewicz space. Other case for $i=2$ and $j=1$ follows similarly. 
In the next theorem, we formulate a necessary and sufficient condition for $X$ to be an $(i, j)$-almost Hurewicz space using $(i, j)$-regular open sets.

Theorem 3.5. Let $\left(X, \mathcal{T}_{1}, \mathcal{T}_{2}\right)$ be a bitopological space. Then $X$ is $(i, j)$-almost Hurewicz if and only if for each sequence $\left(\mathcal{U}_{n}: n \in \mathbb{N}\right)$ of covers of $X$ by $(i, j)$ regular open sets, there exists a sequence $\left(\mathcal{V}_{n}: n \in \mathbb{N}\right)$ such that for each $n \in \mathbb{N}, \mathcal{V}_{n}$ is a finite subset of $\mathcal{U}_{n}$ and for each $x \in X, x \in \bigcup\left\{C l_{\mathcal{T}_{j}}(V): V \in \mathcal{V}_{n}\right\}$ for all but finitely many $n$.

Proof. For the case $i=1$ and $j=2$, necessary part is obvious.

Conversely, assume that $\left(\mathcal{U}_{n}: n \in \mathbb{N}\right)$ is a sequence of $\mathcal{T}_{1}$-open covers of $X$. For each $n \in \mathbb{N}$, define $\mathcal{W}_{n}=\left\{\operatorname{int}_{\mathcal{T}_{1}} C l_{\mathcal{T}_{2}}(U): U \in \mathcal{U}_{n}\right\}$. Then $\left(\mathcal{W}_{n}: n \in \mathbb{N}\right)$ is a sequence of covers of $X$ by $(1,2)$-regular open sets. Therefore, by hypothesis, there exists a sequence $\left(\mathcal{W}_{n}^{\prime}: n \in \mathbb{N}\right)$ such that for each $n \in \mathbb{N}, \mathcal{W}_{n}^{\prime}$ is a finite subset of $\mathcal{W}_{n}$ and for each $x \in X, x \in \bigcup\left\{C l_{\mathcal{T}_{2}}\left(W^{\prime}\right): W^{\prime} \in \mathcal{W}_{n}^{\prime}\right\}$ for all but finitely many $n$. Now for each $n \in \mathbb{N}$ and $W^{\prime} \in \mathcal{W}_{n}^{\prime}$, there exists $U_{W^{\prime}} \in \mathcal{U}_{n}$ such that $W^{\prime}=\operatorname{int}_{\mathcal{T}_{1}} C l_{\mathcal{T}_{2}}\left(U_{W^{\prime}}\right)$. Since $C l_{\mathcal{T}_{2}}\left(U_{W^{\prime}}\right)$ is a $(2,1)$-regular closed set, therefore $C l_{\mathcal{T}_{2}}\left(W^{\prime}\right)=C l_{\mathcal{T}_{2}}\left(U_{W^{\prime}}\right)$. Put $\mathcal{V}_{n}=\left\{U_{W^{\prime}}: W^{\prime} \in \mathcal{W}_{n}^{\prime}\right\}$. For each $n \in \mathbb{N}, \mathcal{V}_{n}$ is a finite subset of $\mathcal{U}_{n}$ and for each $x \in X, x \in \bigcup\left\{C l_{\mathcal{T}_{2}}(V): V \in \mathcal{V}_{n}\right\}$ for all but finitely many $n$. Other case for $i=2$ and $j=1$ follows similarly.

Theorem 3.6. Let $\left(X, \mathcal{T}_{1}, \mathcal{T}_{2}\right)$ and $\left(Y, \mathcal{T}_{1}^{\prime}, \mathcal{T}_{2}^{\prime}\right)$ be two bitopological spaces. Let $X$ be an $(i, j)$-almost Hurewicz space. If $f: X \longrightarrow Y$ is a p-almost continuous onto map, then $Y$ is $(i, j)$-almost Hurewicz.

Proof. Let $i=1$ and $j=2$. Let $\left(\mathcal{U}_{n}: n \in \mathbb{N}\right)$ be a sequence of covers of $Y$ by $(1,2)$-regular open sets. For each $n \in \mathbb{N}$, put $\mathcal{W}_{n}=\left\{f^{-1}(U): U \in \mathcal{U}_{n}\right\}$. Then $\left(\mathcal{W}_{n}: n \in \mathbb{N}\right)$ is a sequence of $\mathcal{T}_{1}$-open covers of $X$. Also as $X$ is $(1,2)$-almost Hurewicz, there exists a sequence $\left(\mathcal{W}_{n}^{\prime}: n \in \mathbb{N}\right)$ such that for each $n \in \mathbb{N}, \mathcal{W}_{n}^{\prime}$ is a finite subset of $\mathcal{W}_{n}$ and for each $x \in X, x \in \bigcup\left\{C l_{\mathcal{T}_{2}}\left(W^{\prime}\right): W^{\prime} \in \mathcal{W}_{n}^{\prime}\right\}$ for all but finitely many $n$. For any $W^{\prime} \in \mathcal{W}_{n}^{\prime}$, choose $U_{W^{\prime}} \in \mathcal{U}_{n}$ such that $W^{\prime}=f^{-1}\left(U_{W^{\prime}}\right)$. For each $n \in \mathbb{N}$, put $\mathcal{V}_{n}=\left\{U_{W^{\prime}}: W^{\prime} \in \mathcal{W}_{n}^{\prime}\right\}$. Then $\left(\mathcal{V}_{n}: n \in \mathbb{N}\right)$ is a sequence such that for any $n \in \mathbb{N}, \mathcal{V}_{n}$ is a finite subset of $\mathcal{U}_{n}$ and for each $y \in Y$, $y \in \bigcup\left\{C l_{\mathcal{T}_{2}^{\prime}}\left(U_{\mathcal{W}^{\prime}}\right): W^{\prime} \in \mathcal{W}_{n}^{\prime}\right\}$ for all but finitely many $n$.

Let $y=f(x) \in Y$. Then there exist $n \in \mathbb{N}$ and $U_{W^{\prime}} \in \mathcal{V}_{n}$ such that $x \in$ $C l_{\mathcal{T}_{2}}\left(f^{-1}\left(U_{W^{\prime}}\right)\right)$. Since $Y \backslash C l_{\mathcal{T}_{2}^{\prime}}\left(U_{W^{\prime}}\right)$ is $(2,1)$-regular open and $f$ is $(2,1)$-almost continuous, so $f^{-1}\left(C l_{\mathcal{T}_{2}^{\prime}}\left(U_{W^{\prime}}\right)\right)$ is $\mathcal{T}_{2}$-closed and $C l_{\mathcal{T}_{2}}\left(f^{-1}\left(U_{W^{\prime}}\right)\right) \subseteq f^{-1}\left(C l_{\mathcal{T}_{2}^{\prime}}\left(U_{W^{\prime}}\right)\right)$. Thus, $y \in C l_{\mathcal{T}_{2}^{\prime}}\left(U_{W^{\prime}}\right)$ so $y \in \bigcup\left\{C l_{\mathcal{T}_{2}^{\prime}}\left(U_{\mathcal{W}^{\prime}}\right): W^{\prime} \in \mathcal{W}_{n}^{\prime}\right\}$ for all but finitely many $n$. Other case for $i=2$ and $j=1$ follows similarly.

Recall that an open cover $\mathcal{U}$ of a topological space $X$ is said be an $\omega$-cover if for each finite subset $F$ of $X$, there exists $U \in \mathcal{U}$ such that $F \subseteq U$ and $X$ is not a member of $\mathcal{U}$. 
Theorem 3.7. Let $\left(X, \mathcal{T}_{1}, \mathcal{T}_{2}\right)$ be a bitopological space. For any $k \in \mathbb{N},\left(X^{k}, \mathcal{T}_{1}^{k}, \mathcal{T}_{2}^{k}\right)$ is $(1,2)$-almost Hurewicz if and only if for each sequence $\left(\mathcal{U}_{n}: n \in \mathbb{N}\right)$ of $\mathcal{T}_{1}-\omega$ covers of $X$, there exists a sequence $\left(\mathcal{V}_{n}: n \in \mathbb{N}\right)$ such that for each $n \in \mathbb{N}, \mathcal{V}_{n}$ is a finite subset of $\mathcal{U}_{n}$ and for every finite subset $F$ of $X, F^{k} \subseteq \bigcup\left\{\left(C l_{\mathcal{I}_{2}}(V)\right)^{k}: V \in \mathcal{V}_{n}\right\}$ for all but finitely many $n$.

Proof. Let $\left(\mathcal{U}_{n}: n \in \mathbb{N}\right)$ be a sequence of $\mathcal{T}_{1}-\omega$-covers of $X$. Let $\left\{N_{m}: m \in \mathbb{N}\right\}$ be a partition of $\mathbb{N}$ such that $N_{m}$ is infinite for each $n \in \mathbb{N}$. For any $m \in \mathbb{N}$ and $i \in N_{m}$, put $\mathcal{U}_{i}^{m}=\left\{U^{m}: U \in \mathcal{U}_{i}\right\}$ then $\left(\mathcal{U}_{i}^{m}: i \in N_{m}\right)$ is a sequence of $\mathcal{T}_{1}^{m}$-open covers of $X^{m}$. By hypothesis, there exists a sequence $\left(\mathcal{V}_{j}^{m}: j \in N_{m}\right)$ such that for each $j \in$ $N_{m}, \mathcal{V}_{j}^{m}$ is a finite subset of $\mathcal{U}_{j}^{m}$ and for each $x \in X^{m}, x \in \bigcup\left\{C l_{\mathcal{T}_{2}^{m}}(V): V \in \mathcal{V}_{j}^{m}\right\}$ for all but finitely many $i$. For every $V \in \mathcal{V}_{j}^{m}$ where $m \in \mathbb{N}, j \in N_{m}$, choose $U_{V} \in \mathcal{U}_{j}$ such that $V=U_{V}^{m}$. Thus, $\left(\mathcal{V}_{n}: n \in \mathbb{N}\right)$ with $\mathcal{V}_{j}=\left\{U_{V}: V \in \mathcal{V}_{j}^{m}\right\}$ is a sequence such that for each $n \in \mathbb{N}, \mathcal{V}_{n}$ is a finite subset of $\mathcal{U}_{n}$. Now let $F=\left\{x_{1}, x_{2}, \ldots, x_{k}\right\}$ be a finite subset of $X$. Since for each $x \in X^{k}, x \in \bigcup C l_{\mathcal{T}_{2}^{k}}\left(\mathcal{V}_{j}^{k}\right)$ for all but finitely many $j$. Therefore, $x^{\prime}=\left(x_{1}, x_{2}, \ldots, x_{k}\right) \in \bigcup C l_{\mathcal{T}_{2}^{k}}\left(\mathcal{V}_{j}^{k}\right)$. So $F^{k} \subseteq \cup C l_{\mathcal{T}_{2}^{k}}\left(\mathcal{V}_{j}^{k}\right)$ for all but finitely many $j$. Since $C l_{\mathcal{T}_{2}^{k}}(V)=C l_{\mathcal{T}_{2}^{k}}\left(U_{V}^{k}\right)=\left(C l_{\mathcal{T}_{2}}\left(U_{V}\right)\right)^{k}$ for some $U_{V} \in \mathcal{V}_{j}$, then $F^{k} \subseteq \bigcup\left\{\left(C l_{\mathcal{T}_{2}}\left(U_{V}\right)\right)^{k}: U_{V} \in \mathcal{V}_{j}\right\}$ for all but finitely many $j$.

Conversely, assume that $\left(\mathcal{U}_{n}: n \in \mathbb{N}\right)$ is a sequence of $\mathcal{T}_{1}^{k}$-open covers of $X^{k}$, where $k$ is fixed number and $\mathcal{U}_{n}=\left\{U_{n m}: m \in I_{n}\right\}$ for each $n$. Suppose $F$ be any finite subset of $X$. So, $F^{k}$ is a finite subset of $X^{k}$. Therefore, for each $n \in \mathbb{N}$, there exists a finite subset $I_{n}^{F} \subseteq I_{n}$ such that $F^{k} \subseteq \bigcup_{m \in I_{n}^{F}} U_{n m}$. So there exists a $\mathcal{T}_{1}$-open set $V_{F}$ such that $F \subseteq V_{F}$ and $F^{k} \subseteq V_{F}^{k} \subseteq \bigcup_{m \in I_{n}^{F}} U_{n m}$. Hence, $\mathcal{V}_{n}=\left\{V_{F}: F\right.$ is finite subset of $\left.X\right\}$ is a $\mathcal{T}_{1}-\omega$-open cover of $X$. By hypothesis, there exists a sequence $\left(\mathcal{W}_{n}: n \in \mathbb{N}\right)$ such that for each $n \in \mathbb{N}, \mathcal{W}_{n}$ is a finite subset of $\mathcal{U}_{n}$ and for any finite subset $T$ of $X, T^{k} \subseteq \bigcup\left\{\left(C l_{\mathcal{T}_{2}}(W)\right)^{k}: W \in \mathcal{W}_{n}\right\}$ for all but finitely many $n$. Now for each $n \in \mathbb{N}$ let $\mathcal{W}_{n}=\left\{V_{F_{j}}: j \in J_{n}\right\}$ where $J_{n}$ is a finite index set. Further, assume that $\mathcal{K}_{n}=\left\{U_{n m}: m \in H_{n}\right\}$ where $H_{n}=\left\{m \in I_{n}: m \in I_{n}^{F_{j}}, j \in J_{n}\right\}$ then $\mathcal{K}_{n}$ is a finite subset of $\mathcal{U}_{n}$ and for each $x=\left(x_{1}, x_{2}, \ldots, x_{k}\right) \in X^{k}, x \in \bigcup\left\{C l_{\mathcal{T}_{2}^{k}}\left(U_{n m}\right): m \in H_{n}\right\}$ for all but finitely many $n$.

Theorem 3.8. Let $\left(X, \mathcal{T}_{1}, \mathcal{T}_{2}\right)$ be a bitopological space and $A \subseteq X$. If the subspace $\left(A, \mathcal{T}_{1_{A}}, \mathcal{T}_{2_{A}}\right)$ is $(i, j)$-almost Hurewicz, then for every sequence of covers $\left(\mathcal{U}_{n}: n \in\right.$ $\mathbb{N})$ of $A$ by $\mathcal{T}_{i}$-open sets in $X$, there exists a sequence $\left(\mathcal{V}_{n}: n \in \mathbb{N}\right)$ such that for each $n \in \mathbb{N}, \mathcal{V}_{n}$ is a finite subset of $\mathcal{U}_{n}$ and $A \subseteq \bigcup_{n} \bigcup\left\{C l_{\mathcal{T}_{j}}(V): V \in \mathcal{V}_{n}\right\}$.

Proof. Let $i=1$ and $j=2$. Now suppose $\left(\mathcal{U}_{n}: n \in \mathbb{N}\right)$ be a sequence of covers of $A$ by $\mathcal{T}_{1}$-open sets in $X$. Define $\mathcal{U}_{n}^{*}=\left\{A \cap U: U \in \mathcal{U}_{n}\right\}$. Thus, $\left(\mathcal{U}_{n}^{*}: n \in \mathbb{N}\right)$ is a sequence of $\mathcal{T}_{1_{A}}$-open covers of $A$. Therefore, by hypothesis, there exists a sequence $\left(\mathcal{V}_{n}^{*}: n \in \mathbb{N}\right)$ such that for each $n \in \mathbb{N}, \mathcal{V}_{n}^{*}$ is a finite subset of $\mathcal{U}_{n}^{*}$ and for each $a \in A, a \in \bigcup\left\{C l_{\mathcal{T}_{2_{A}}}(V): V \in \mathcal{V}_{n}^{*}\right\}$ for all but finitely many $n$. Note that for each $n \in \mathbb{N}$ and each $V \in \mathcal{V}_{n}^{*}$, there is a set $U_{V} \in \mathcal{U}_{n}$ such that $V=A \cap U_{V}$. Therefore, 
for each $n \in \mathbb{N}, \mathcal{V}_{n}=\left\{U_{V}: V \in \mathcal{V}_{n}^{*}\right\}$ is a finite subset of $\mathcal{U}_{n}$ and for each $V \in \mathcal{V}_{n}^{*}$, $C l_{\mathcal{T}_{2_{A}}}(V) \subseteq C l_{\mathcal{T}_{2}}\left(U_{V}\right)$. Hence, $A \subseteq \bigcup_{n} \bigcup\left\{C l_{\mathcal{T}_{2}}\left(U_{V}\right): U_{V} \in \mathcal{V}_{n}\right\}$. Other case for $i=2$ and $j=1$ follows similarly.

Theorem 3.9. Let $\left(X, \mathcal{T}_{1}, \mathcal{T}_{2}\right)$ be a bitopological space and $A \subseteq X$ be $\mathcal{T}_{j}$-open. Suppose that for every sequence of covers $\left(\mathcal{U}_{n}: n \in \mathbb{N}\right)$ of $A$ by $\mathcal{T}_{i}$-open sets in $X$, there exists a sequence $\left(\mathcal{V}_{n}: n \in \mathbb{N}\right)$ such that for each $n \in \mathbb{N}, \mathcal{V}_{n}$ is a finite subset of $\mathcal{U}_{n}$ and $A \subseteq \bigcup\left\{C l_{\mathcal{T}_{j}}(V): V \in \mathcal{V}_{n}\right\}$ for all but finitely many $n$. Then the subspace $\left(A, \mathcal{T}_{1_{A}}, \mathcal{T}_{2_{A}}\right)$ is $(i, j)$-almost Hurewicz.

Proof. Let $i=1, j=2$. Let $\left(\mathcal{U}_{n}: n \in \mathbb{N}\right)$ be a sequence of covers of $A$ by $\mathcal{T}_{1_{A}}$-open sets in $A$. For each $n \in \mathbb{N}$ and $U \in \mathcal{U}_{n}$, there exists $V_{U} \in \mathcal{T}_{1}$ such that $U=A \cap V_{U}$. Therefore, $\left(\mathcal{V}_{n}: n \in \mathbb{N}\right)$ is a sequence of covers of $A$ by $\mathcal{T}_{1}$-open sets in $X$, where $\mathcal{V}_{n}=\left\{V_{U}: U \in \mathcal{U}_{n}\right\}$. By hypothesis, there is a sequence $\left(\mathcal{V}_{n}^{\prime}: n \in \mathbb{N}\right)$ such that for each $n \in \mathbb{N}, \mathcal{V}_{n}^{\prime}$ is a finite subset of $\mathcal{V}_{n}$ and $A \subseteq \bigcup\left\{C l_{\mathcal{T}_{2}}\left(V_{U}\right): V_{U} \in \mathcal{V}_{n}^{\prime}\right\}$ for all but finitely $n$. Now for each $n \in \mathbb{N}$, put $\mathcal{U}_{n}^{\prime}=\left\{A \cap V_{U}: V_{U} \in \mathcal{V}_{n}^{\prime}\right\}$. Then $\mathcal{U}_{n}^{\prime}$ is a finite subset of $\mathcal{U}_{n}$. Note that for each $n \in \mathbb{N}$ and each $U \in \mathcal{U}_{n}^{\prime}$, $C l_{\mathcal{T}_{2_{A}}}(U)=C l_{\mathcal{T}_{2_{A}}}\left(V_{U} \cap A\right)=C l_{\mathcal{T}_{2}}\left(V_{U} \cap A\right) \cap A \subseteq C l_{\mathcal{T}_{2}}\left(V_{U}\right) \cap A$. Also, since $A$ is $\mathcal{T}_{2}$-open, therefore $C l_{\mathcal{T}_{2}}\left(V_{U}\right) \cap A \subseteq C l_{\mathcal{T}_{2}}\left(V_{U} \cap A\right)$. Thus, $C l_{\mathcal{T}_{2_{A}}}(U)=C l_{\mathcal{T}_{2}}\left(V_{U}\right) \cap A$. Hence, $A \subseteq \bigcup\left\{C l_{\mathcal{T}_{2_{A}}}(U): U \in \mathcal{U}_{n}^{\prime}\right\}$ for all but finitely many $n$. Other case for $i=2$ and $j=1$ follows similarly.

Theorem 3.10. Let $\left(X, \mathcal{T}_{1}, \mathcal{T}_{2}\right)$ be an $(i, j)$-almost Hurewicz space and $A$ be $\mathcal{T}_{i}$ closed, $\mathcal{T}_{j}$ - open subset of $X$. Then $A$ is also $(i, j)$-almost Hurewicz space.

Proof. Let $i=1$ and $j=2$. Suppose $\left(\mathcal{U}_{n}: n \in \mathbb{N}\right)$ be a sequence of covers of $A$ by $\mathcal{T}_{1}$-open sets in $X$. Now for each $n \in \mathbb{N}, \mathcal{V}_{n}=\mathcal{U}_{n} \cup\{X \backslash A\}$ is a $\mathcal{T}_{1}$-open cover of $X$. Therefore, by hypothesis, there is a sequence $\left(\mathcal{V}_{n}^{\prime}: n \in \mathbb{N}\right)$ such that for each $n \in \mathbb{N}$, $\mathcal{V}_{n}^{\prime}$ is a finite subset of $\mathcal{V}_{n}$ and for each $x \in X, x \in \bigcup\left\{C l_{\mathcal{T}_{2}}(V): V \in \mathcal{V}_{n}^{\prime}\right\}$ for all but finitely many $n$. Now for every $n \in \mathbb{N}$, choose $\mathcal{U}_{n}^{\prime}=\left\{V \in \mathcal{V}_{n}^{\prime}: V \neq(X \backslash A)\right\}$. Then $\mathcal{U}_{n}^{\prime}$ is a finite subset of $\mathcal{U}_{n}$. Also note that $A$ is $\mathcal{T}_{2}$-open with $A \subseteq \bigcup\left\{C l_{\mathcal{T}_{2}}(V)\right.$ : $\left.V \in \mathcal{U}_{n}^{\prime}\right\}$ for all but finitely many $n$. Hence, by Theorem 3.9, $A$ is (1,2)-almost Hurewicz. Other case for $i=2$ and $j=1$ follows similarly.

Theorem 3.11. Let $\left(X, \mathcal{T}_{1}, \mathcal{T}_{2}\right)$ and $\left(Y, \mathcal{T}_{1}^{\prime}, \mathcal{T}_{2}^{\prime}\right)$ be bitopological spaces such that $f$ : $X \longrightarrow Y$ is $(j, i)$-preopen and perfect map. If $\left(Y, \mathcal{T}_{1}^{\prime}, \mathcal{T}_{2}^{\prime}\right)$ is $(i, j)$-almost Hurewicz, then $\left(X, \mathcal{T}_{1}, \mathcal{T}_{2}\right)$ is also $(i, j)$-almost Hurewicz.

Proof. Let $i=1$ and $j=2$. Now assume that $\left(\mathcal{U}_{n}: n \in \mathbb{N}\right)$ be a sequence of $\mathcal{T}_{1}$ open covers of $X$. Then for each $y \in Y$ and $n \in \mathbb{N}$, there exists a finite subfamily $\mathcal{U}_{n_{y}}$ of $\mathcal{U}_{n}$ such that $f^{-1}(y) \subseteq \cup \mathcal{U}_{n_{y}}$. Put $U_{n_{y}}=\bigcup \mathcal{U}_{n_{y}}$. Note that for each $n \in \mathbb{N}, V_{n_{y}}=Y \backslash f\left(X \backslash U_{n_{y}}\right)$ is a $\mathcal{T}_{1}^{\prime}$ - open neighbourhood of $y$. Therefore, take $\mathcal{V}_{n}=\left\{V_{n_{y}}: y \in Y\right\}$. Thus, $\left(\mathcal{V}_{n}: n \in \mathbb{N}\right)$ is a sequence of $\mathcal{T}_{1}^{\prime}$ - open covers of $Y$. Therefore, there exists a sequence $\left(\mathcal{V}_{n}^{\prime}: n \in \mathbb{N}\right)$ such that for each $n \in \mathbb{N}$, 
H.V.S. CHAUHAN, B. SINGH

$\mathcal{V}_{n}^{\prime}$ is a finite subset of $\mathcal{V}_{n}$ and for each $y \in Y, y \in \bigcup\left\{C l_{\mathcal{T}_{2}^{\prime}}(V): V \in \mathcal{V}_{n}^{\prime}\right\}$ for all but finitely many $n$. Now for each $n \in \mathbb{N}$, choose $\mathcal{V}_{n}^{\prime}=\left\{V_{n_{y_{i}}}: i \leq n^{\prime}\right\}$. Corresponding to each $\mathcal{V}_{n}^{\prime}$, choose $\mathcal{U}_{n}^{\prime}=\left\{U_{n_{y_{i}}}: i \leq n^{\prime}\right\}=\left\{\bigcup \mathcal{U}_{n_{y_{i}}}: i \leq n^{\prime}\right\}$. Therefore, $\mathcal{U}_{n}^{\prime}$ is a finite subset of $\mathcal{U}_{n}$. As $f$ is $(2,1)$-preopen, therefore for each $x \in X, x \in f^{-1}\left(\bigcup\left\{C l_{\mathcal{T}_{2}^{\prime}}\left(V_{n_{y_{i}}}\right): i \leq n^{\prime}\right\}\right)$ for all but finitely many $n$. Since $f^{-1}\left(\bigcup\left\{C l_{\mathcal{T}_{2}^{\prime}}\left(V_{n_{y_{i}}}\right): i \leq n^{\prime}\right\}\right) \subseteq \bigcup\left\{C l_{\mathcal{T}_{2}}\left(f^{-1}\left(V_{n_{y_{i}}}\right)\right): i \leq n^{\prime}\right\} \subseteq \bigcup\left\{C l_{\mathcal{T}_{2}}\left(U_{n_{y_{i}}}\right): i \leq\right.$ $\left.n^{\prime}\right\}=\bigcup\left\{C l_{\mathcal{T}_{2}}(U): U \in \mathcal{U}_{n}^{\prime}\right\}$, then $x \in \bigcup\left\{C l_{\mathcal{T}_{2}}(U): U \in \mathcal{U}_{n}^{\prime}\right\}$ for all but finitely many $n$. Hence, $X$ is $(1,2)$-almost Hurewicz. Other case for $i=2$ and $j=1$ follows similarly.

Theorem 3.12. Let $\left(X, \mathcal{T}_{1}, \mathcal{T}_{2}\right)$ and $\left(Y, \mathcal{T}_{1}^{\prime}, \mathcal{T}_{2}^{\prime}\right)$ be bitopological spaces such that $X$ is $(i, j)$-almost Hurewicz and $Y$ is d-compact. Then $X \times Y$ is also $(i, j)$-almost Hurewicz.

Proof. Let $i=1$ and $j=2$. The projection map $p_{X}: X \times Y \longrightarrow X$ is perfect as $Y$ is $d$-compact. Further since $p_{X}$ is $d$-open, therefore it is also $(2,1)$-preopen. Using Theorem 3.11, $X \times Y$ is $(1,2)$ - almost Hurewicz. Other case for $i=2$ and $j=1$ follows similarly.

\section{REFERENCES}

[1] Datta, M.C., Projective bitopological spaces II, J. Aust. Math. Soc., 14 (1972), 119 - 128.

[2] Dvalishvili, B.P., Bitopological spaces: Theory, relations with generalized algebraic structures and applications, North Holland Mathematics studiesm, Elsevier science B.V., Amsterdam, (2005).

[3] Engelking, R., General Topology, Heldermann Verlag, Berlin, (1989).

[4] Eysen, A., Özçă̆, S., Weaker forms of the Menger property in bitopological spaces, Quaest. Math., 41(7) (2018), $877-888$.

[5] Hurewicz, W., Über die verallgemeinerung des Borelschen Theorems, Math. Z., 24 (1925), $401-425$.

[6] Hurewicz, W., Über Folgen Stetiger Funktionen, Fund. Math., 9 (1927), 193 - 204.

[7] Jelić, M., Feebly p-continuous mappings, $V$ International meeting on topology in Italy (Italian) (Lecce,1990/Otranto, 1990); Rend. Circ. Mat. Palermo, 24 (2) (1990), 387 - 395.

[8] Khan, M.D., Sabah, A., Selection principles and covering properties in bitopological spaces, Applied General Topology, 21(1) (2020), $159-169$.

[9] Khedr, F.H., Alshibani, A.M., On pairwise super continuous mappings in bitopological spaces, Int. J. Math. Math. Sci., 14(4) (1991), $715-722$.

[10] Kočinac, Lj.D.R., The Pixley-Roy topology and selection principles, Questions and Answers in General Topology, 19(2) (2001), $219-225$.

[11] Kočinac, Lj.D.R., The Reznichenko property and the Pytkeev property in hyperspaces, Acta Mathematica Hungarica, 107(3) (2005), 225 - 233

[12] Kočinac, Lj.D.R., Özçă̆, S., Versions of separability in bitopological spaces, Topology and its Applications 158 (2011), $1471-1477$.

[13] Kočinac, Lj.D.R., Özçă̆, S., Bitopological spaces and selection principles, Proceedings ICTA 2011, Cambridge Scientific Publishers, (2012), 243 - 255.

[14] Lyakhovets, D., Osipov, A.V., Selection principles and games in bitopological function spaces, Filomat, 33(14) (2019), 4535 - 4540. 
[15] Maheshwari, S.N., Prasad, R. Semi open sets and semi continuous function in bitopological spaces, Math. Notes 26 (1977-78), $29-37$.

[16] Menger, K., Einige Überdeckungssätze der Punktmengenlehre, Sitzungsber. Österr. Adad. Wiss. Math.-Naturwiss. Kl. Abt IIa, 133 (1924), $421-444$

[17] Özçă̆, S., Bornologies and bitopological function spaces, Filomat 27(7) (2013), $1345-1349$.

[18] Özçă̆, S., Selective bitopological version of separability, Topology and its Applications, 201 (2016), $403-413$.

[19] Özçă̆, S., Eysen, A.E., Almost Menger property in bitopological spaces, Ukrainian Math. J., 68(6) (2016), $950-958$.

[20] Rothberger, F., Eine Verscharfung der Eigenschaft C, Fund. Math. 30 (1938), 50 - 55.

[21] Sakai, M., The weak Hurewicz property of Pixley-Roy hyperspaces, Topology and its Applications, 160(18) (2013), $2531-2537$.

[22] Scheepers, M., Combinatorics of open covers I: Ramsey theory, Topology Appl. 69 (1996), $31-62$.

[23] Singal, A.R., Arya, S.P., On pairwise almost regular spaces, Glasnik Math. 26(6) (1971), $335-343$.

[24] Song, Y.K., Li, R., The almost Hurewicz spaces, Questions and Answers in General Topology 31 (2013), $131-136$.

[25] Song, Y.K., Li, R., On weakly Hurewicz spaces, Filomat 29(4) (2015), 667 - 671. 